\title{
Intermediate behavior of Kerr tails
}

\author{
Anıl Zenginoğlu · Gaurav Khanna · Lior \\ M. Burko
}

the date of receipt and acceptance should be inserted later

\begin{abstract}
The numerical investigation of wave propagation in the asymptotic domain of Kerr spacetime has only recently been possible thanks to the construction of suitable hyperboloidal coordinates. The asymptotics revealed an apparent puzzle in the decay rates of scalar fields: the late-time rates seemed to depend on whether finite distance observers are in the strong field domain or far away from the rotating black hole, an apparent phenomenon dubbed 'splitting.' We discuss far-field 'splitting' in the full field and near-horizon 'splitting' in certain projected modes using horizonpenetrating, hyperboloidal coordinates. For either case we propose an explanation to the cause of the 'splitting' behavior, and we determine uniquely decay rates that previous studies found to be ambiguous or immeasurable. The far-field 'splitting' is explained by competition between projected modes. The near-horizon 'splitting' is due to excitation of lower multipole modes that back excite the multipole mode for which 'splitting' is observed. In both cases 'splitting' is an intermediate effect, such that asymptotically in time strong field rates are valid at all finite distances. At any finite time, however, there are three domains with different decay rates whose boundaries move outwards during evolution. We then propose a formula for the decay rate of tails that takes into account the inter-mode excitation effect that we study.
\end{abstract}

Keywords Black hole perturbation theory - Wave equations · Tail decay . Hyperboloidal compactification

\author{
A. Zenginoğlu \\ Theoretical Astrophysics, California Institute of Technology, Pasadena, California USA \\ G. Khanna \\ Department of Physics, University of Massachusetts, Dartmouth, Massachusetts USA
}

L. M. Burko

Université d'Orléans, Observatoire des Sciences de l'Univers en Region Centre, LPC2E Campus CNRS, 45071 Orléans, France

and Department of Physics, Chemistry, and Mathematics, Alabama A\&M University, Normal, Alabama 35762, USA

and Theiss Research, La Jolla, California 92037, USA 


\section{Introduction}

The response of a black hole to generic perturbations has been an active topic of interest since the study of Regge and Wheeler in 1957 [1]. It is now well-known that black hole perturbations satisfy wave equations. Generic solutions to such wave equations consist of three stages: an initial transient during which the evolution depends on details of initial data; exponentially decaying oscillations called quasinormal mode ringing; and a late-time polynomial decay dubbed the Price tail. In this paper, we focus on the late-time behavior during which the field decays as $t^{n}$, where $t$ denotes time measured by stationary observers and $n$ denotes the decay rate ([2]), and specifically distinguish between intermediate albeit late time, and asymptotically late-time behaviors.

The historical analyses of the Kerr decay rates are based on asymptotic expansion methods, and have also been studied extensively. Theoretical work has provided the general expected decay rates of perturbations in Kerr spacetime [3, 4, 5, 6,7]. These rates have also been computed numerically [8,9,10,11,12]. Today, our understanding of late-time Kerr decay rates of spherical harmonic $Y_{\ell m}$ modes of the scalar field includes as an essential element the distinction between initial modes and projected modes generated by coupling. Denoting the initial mode number by $\ell^{\prime}$ and the projected mode number by $\ell$, and focusing on azimuthal $(m=0)$ modes, the asymptotic finite-distance decay rates of projected modes was found to be given by $n^{R}=-\left(\ell^{\prime}+\ell+1\right)$ for $\ell<\ell^{\prime}$ and $n^{R}=-\left(\ell^{\prime}+\ell+3\right)$ for $\ell \geq \ell^{\prime}$ where $R$ is a finite value for the Boyer-Lindquist radial coordinate. Decay rates along null infinity differ from those at finite distances [13]. The rates along null infinity of Kerr spacetime predicted by Hod $([5]) n^{\mathscr{I}^{+}}=-(\ell+2)$ for $\ell \geq \ell^{\prime}$ and $n^{\mathscr{I}^{+}}=-\ell^{\prime}$ for $\ell \leq \ell^{\prime}-2$. In summary:

$$
n^{R}=\left\{\begin{array}{l}
-\left(\ell^{\prime}+\ell+1\right) \text { for } \quad \ell<\ell^{\prime}, \\
-\left(\ell^{\prime}+\ell+3\right) \text { for } \quad \ell \geq \ell^{\prime}
\end{array} \quad n^{\mathscr{I}^{+}}= \begin{cases}-\ell^{\prime} & \text { for } \quad \ell \leq \ell^{\prime}-2 \\
-(\ell+2) & \text { for } \quad \ell \geq \ell^{\prime}\end{cases}\right.
$$

We revisit this formula in Section 3 with particular focus on the cases $\ell^{\prime}=\ell$, and our results suggest to us that Eq. (1) needs to be revised to include high-order couplings that are not captured in Eq. (1). The spherical harmonic modes $(\ell, m)$ in this work are the same as those in earlier works that use Boyer-Lindquist coordinates [11,12,14] and other coordinates in the same equivalence-class [15,16]. Thus, the same late-time decay rates are observed for each mode.

Until recently, the numerical study of Kerr tails in the literature has exclusively focused on finite distance rates even though the null infinity rates are arguably more interesting for observers at astronomical distances $([17,18])$. Null infinity decay rates in Schwarzschild spacetime have been studied with the characteristic method as early as 1994 ([13]). The characteristic method, however, is difficult to extend to Kerr spacetime $([19,20,21])$. It is due to this technical obstacle that Kerr tails in the asymptotic domain have not been studied numerically.

This problem has recently been resolved with the general construction of hyperboloidal coordinates for asymptotically flat black hole spacetimes ([22]). These coordinates provide numerical access to the asymptotic domain and resolve the outer 
boundary problem in an efficient way. Recent hyperboloidal evolutions ([14, 15, 16, 23]) confirmed the theoretically predicted decay rates of projected modes at null infinity.

The hyperboloidal evolutions revealed an apparently puzzling behavior for the rates in the asymptotic spatial domain. The rates at finite distances far away from the black hole and at finite times appeared to deviate from the rates both near the black hole (including the event horizon), and along null infinity. This observation requires some explanation. In Schwarzschild spacetime, the local (in time) decay rates at finite distances depend on the distance to the black hole. For example, the dominant $\ell^{\prime}=\ell=0$ mode decays along null infinity with a power of -2 , and at finite distances with -3 , asymptotically in time. Therefore, at any finite time, one expects that the finite distance rates vary monotonously between -2 and -3 . This expectation is in accordance with theoretical predictions (see Eq. (5) and [24,25]) and is confirmed by numerical studies ([17, 18]).

A similar distance-dependence for the full field is observed in Kerr spacetime for $\ell^{\prime}<4$, but for initial data with $\ell^{\prime}=4$ an anomalous behavior appears. Instead of a monotonous transition between the null infinity rate of -4 and the finite distance rate of -5 , there are apparently far away observers for whom the decay rate seems to approach a value smaller than -5 ([14]). A more detailed study of tail decay rates by Rácz and Tóth in [15] provided independent evidence for such anomalous behavior. They suggested that certain projected modes have different local decay rates close to the horizon and far away from it, which they referred to as 'splitting.' They also found that the 'splitting' depends upon the value of the azimuthal mode number $m$. Numerical computations in 3D by Jasiulek showed that 'splitting' is robust ([16]). These studies pointed out that longer time evolutions are needed, suggesting that 'splitting' may not be an asymptotic but rather an intermediate phenomenon. Rácz and Tóth in [15] referred to certain decay rates as "ambiguous," indicating that they were unable, because of the "splitting' phenomenon they observed, to determine uniquely the value of the decay rate $n$. More recently, Harms, Bernuzzi, and Bruegmann [26] revisited this question for scalar, electromagnetic, and gravitational perturbations and reported on similar 'splitting' phenomena. They determined some of the rates that [15] listed as "ambiguous" but did not provide rates for all of them.

In this paper, we perform careful evolutions that are nearly an order-of-magnitude longer than previous works (on the order of $10^{4} M$ where $M$ denotes the mass of the Kerr black hole), to study the two types of 'splitting' discussed in the literature: 'splitting' in the full field ([14]) and 'splitting' in certain projected modes ([15, 16, 26]). Our long evolutions, enabled by the advances in numerical technology, allow us to explain the origin of the two kinds of 'splitting' phenomena, conclude that 'splitting' does not exist in the asymptotically late-time decay rate but only as an intermediate feature of the fields evolution, and also determine uniquely all the decay rates that have been found previously to be ambiguous or immeasurable. We emphasize that our evolutions were just as long as needed to determine the new results: shorter evolutions might indeed lead one to conclude that some decay rates are ambiguous

We show that the two 'splitting' phenomena arise from different mechanisms and argue that, asymptotically in time, the strong field decay rates are valid at all finite distances, such that there is no 'splitting' in the asymptotic regime. The full-field 
'splitting' has been explained before, yet we discuss it here again for the completeness of the presentation. 'Splitting' in the full field is nearly-trivially explained as a competition between the constituent multipole modes, such that multipole modes which dominate at early times become eventually sub-dominant. 'Splitting' in projected modes involves a more intricate mechanism: it appears as an intermediate behavior of decay rates from excitation of lower multipole modes that back excite the higher mode in question. Both types of 'splitting' disappear asymptotically. 'Splitting' in the projected modes as an intermediate phenomenon is still interesting and important though, as at any given late time there are three domains observed with different local decay rates for certain projected modes. The boundaries between these domains move to larger distances during evolution.

Our study requires integration into very late times. The technological advances that allowed for such long evolutions may be relevant also for other interesting problems. For example, the late-time decay of scalar fields is related to the violation of the Huygens' principle in black hole spacetimes and therefore carries theoretical interest. Its presence indicates that waves not only propagate on the light cone of the initial perturbation, but also within the light cone. Such propagation is partly responsible for the late-time polynomial decay, also called the tail. The tail piece of the solution is very small, and unlikely to be directly detectable. One may therefore argue that it is not interesting from an astrophysical point of view. However, there are astrophysical problems where the accurate computation of the tail decay is important.

One specific astrophysical problem where one needs to numerically compute the late-time tail solution is related to the inspiral of a small black hole into a supermassive one. The self-force that acts on such a small black hole can be described by a time integral into the infinite past of the retarded Green function that describes perturbations of the supermassive black hole [27,28]. The computation of such black hole Green functions at a given base point can be performed by numerically integrating wave equations with vanishing initial data and a narrow Gaussian source centered at the given base point approximating a Dirac distribution [29]. These computations must be performed into late times where the tail effect dominates because the selfforce calculation includes a half-infinite time integral of the Green function [30]. Therefore accurate computations of late-time wave solutions are important for a theoretical understanding of wave propagation and for the development of numerical tools to tackle astrophysical problems.

The organization of this paper is as follows. In Section 2 we describe the numerical methods that we use, specifically the hyperboloidal compactification of the horizon and of infinity in Section 2.1 and the numerical implementation in Section 2.2. In Section 3 we describe our results for the far-field 3.1) and the (near-field 3.2. cases. We discuss our results in Section 4 


\section{Methods}

2.1 Hyperboloidal compactification of the horizon and of infinity

A technical new development in this paper is the application of the hyperboloidal layer method from [31] to the construction of horizon-penetrating, hyperboloidal coordinates in Kerr spacetime. The causal behavior of the slicing is similar to the slicings of [15,16] with the difference that standard Boyer-Lindquist coordinates can be used in a compact domain. Our coordinates extend those in [32] to become horizonpenetrating. Similar coordinates have been constructed previously for Schwarzschild spacetime in [33].

Denoting the tortoise coordinate based on the Boyer-Lindquist representation of the Kerr metric as $r_{*}$, we map the infinite domain $r_{*} \in(-\infty, \infty)$ to a finite domain $\rho \in[-S, S]$ using the spatial compactification

$$
r_{*}=\frac{\rho}{\Omega}, \quad \text { with } \quad \Omega=1-\left(\frac{|\rho|-\mathrm{R}}{\mathrm{S}-\mathrm{R}}\right)^{4} \Theta(|\rho|-\mathrm{R}),
$$

where $\Theta$ denotes the step function and $R$ the location of the interface. The function $\Omega$ vanishes at $\rho= \pm S$ with a nonzero gradient. It is unity for $-R<\rho<R$ and sufficiently smooth at the interfaces $\rho= \pm R$. To avoid loss of resolution for outgoing waves near infinity, we combine the spatial compactification with a suitable time transformation introducing a new time coordinate $\tau=t \pm h\left(r_{*}\right)$. The function $h\left(r_{*}\right)$ is called the height function.

The Kerr metric in Boyer-Lindquist coordinates is asymptotically Schwarzschild in standard Schwarzschild coordinates. Therefore, the leading order form of in- and outgoing null rays can be written as $t \pm r_{*}$. The height function is chosen such that the time function $\tau$ satisfies the condition $t \pm r_{*}=\tau \pm \rho$. Combined with the spatial compactification (2) we get

$$
h(\rho)=\frac{\rho}{\Omega}-\rho .
$$

The choices (2) and (3) fully determine the coordinates. Note that only the asymptotic form of the in- and outgoing null surfaces goes into the hyperboloidal transformation.

\subsection{Numerical implementation}

We study local decay rates by solving the scalar wave equation, $\square \phi=0$, for a rescaled variable $r \phi$, using hyperboloidal layers. Our numerical simulations are performed with a modified version of the time-domain evolution code presented in [12]. The code is an explicit, hyperbolic-PDE solver in $(2+1) \mathrm{D}$ that uses the Lax-Wendroff finite-difference evolution scheme. The numerical results have low truncation error due to high-order differencing in the angular direction and high-precision (quadruple or octal) floating-point operations. The code is parallelized via message passing interface using the standard domain-decomposition approach on the radial coordinate grid. The main modification of the code for this paper is the implementation of hyperboloidal layers as discussed in the previous section. 
Typical grid resolutions used in this work are $M / 64$ in the radial and $\pi / 48$ in the angular direction. We set $M=1$ and $a / M=0.995$. The domain size in the compactified $\rho$-coordinate is $[-100,100] M$ with the layer interfaces at $R= \pm 28 M$. The time-step is set to $M / 128$ as dictated by the Courant condition for stability of the numerical evolution scheme. The compact-supported, non-stationary, initial data for the scalar field is chosen to be a truncated Gaussian wave packet of width $4 M$ and variable center location. This corresponds to type 1 initial data as classified in [15].

\section{Results}

We investigate the decay rates for all $\ell^{\prime} \leq 5$, specializing to vanishing azimuthal number, $m=0$. We study intermediate decay rates for both the projected modes and the full field measured by various observers using the local decay rate $n^{\text {obs }}(t)$ defined as $([34])$

$$
n^{\mathrm{obs}}:=\frac{d \ln \left|\phi\left(t, r^{\mathrm{obs}}\right)\right|}{d \ln t}
$$

We then define the asymptotic decay rate as $n_{\infty}^{\text {obs }}:=\lim _{t \rightarrow \infty} n^{\text {obs }}$. Note that the local and asymptotic decay rates are invariant under the transformations in the hyperboloidal layers because the timelike Killing field is left invariant by the hyperboloidal time transformation.

\subsection{Far-field}

\subsubsection{Local rates of projected modes}

The intermediate decay rates, as defined in (4). for projected modes with initial data mode $\ell^{\prime} \leq 5$ measured by far-field observers ranging from null infinity to about $100 M$ are shown in Fig. 1 with the center of the Gaussian initial perturbation located at $r_{*}=25 \mathrm{M}$. The decay rates are monotonous; no 'splitting' occurs.

It is instructive to contrast Fig. 1 to observations in Schwarzschild spacetime. The observer dependence and the intermediate behavior of local decay rates are simple there. An initial pure spherical harmonic stays a pure spherical harmonic during evolution $\left(\ell=\ell^{\prime}\right)$ and the decay rates are given as $n_{\infty}^{i^{+}}=-(2 \ell+3)$ at timelike infinity and as $n_{\infty}^{\mathscr{I}}=-(\ell+2)$ at null infinity [2,13]. During the intermediate decay there is a monotonous transition depending on the location of the observer $([17,18])$.

In Kerr spacetime, there is no geometric notion of a pure multipole because of the lack of spherical symmetry of the background spacetime: the scalar spherical harmonics are not eigenfunctions of the Laplace operator, and this leads to coupling between multipoles. Nevertheless, we observe a similar intermediate behavior in the projected modes for far field observers $(r>100 M)$ in Fig. 1

The observer-dependence in these plots can be understood by considering the description of pointwise decay estimates suggested in Schwarzschild spacetime for 

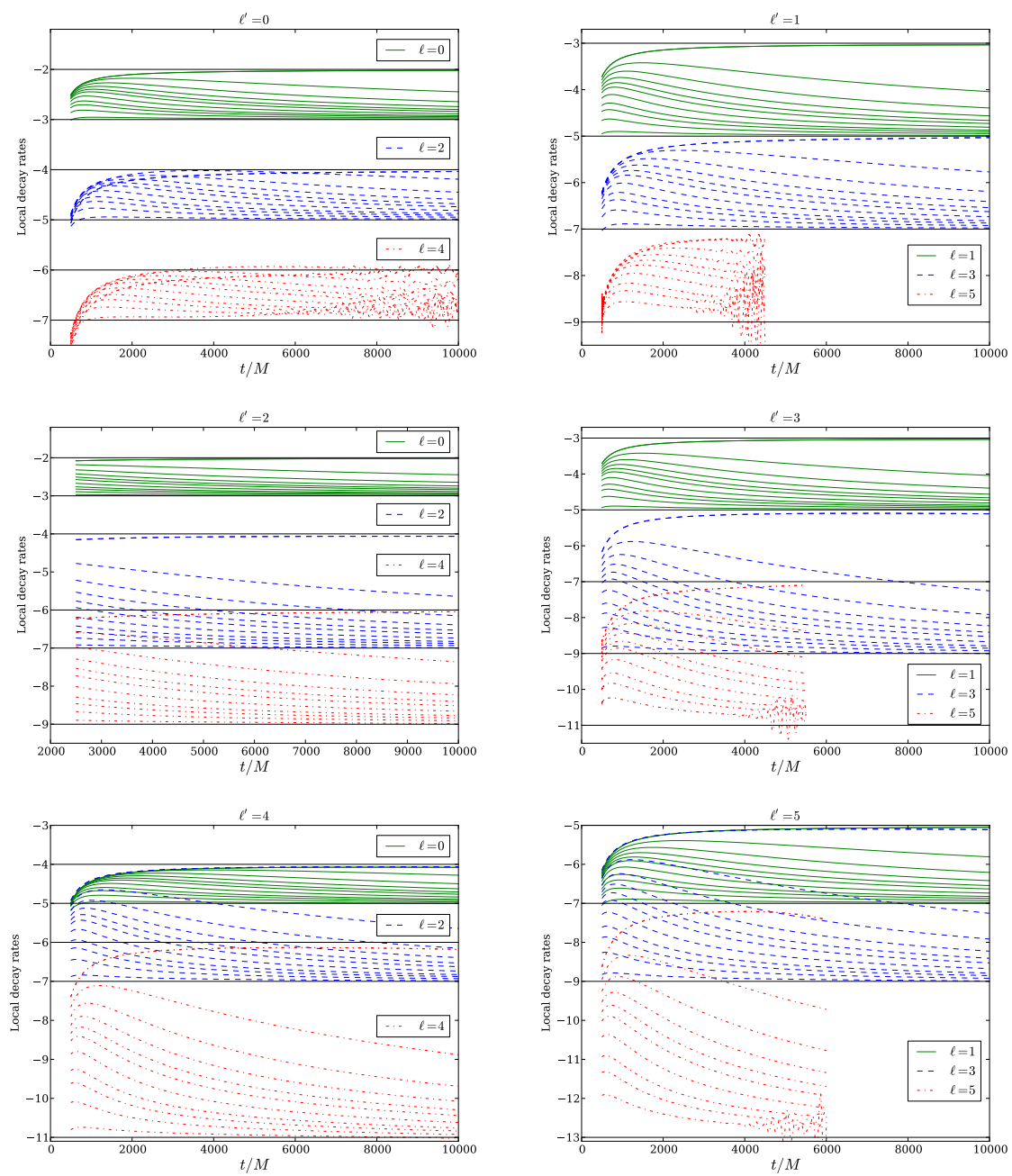

Fig. 1 Local decay rates for the evolution of even initial modes $\ell^{\prime}=0,2,4$ on the left panels and odd initial modes $\ell^{\prime}=1,3,5$ on the right panels, for a family of far-field observers ranging from null infinity to about $100 M$ successively closer to the Kerr hole from top to bottom for each projection. The initial data is a Gaussian with compact support centered at $r_{*}=25 \mathrm{M}$. The late-time values are consistent with the predictions in the published literature. No 'splitting' is observed in the far field $(r \geq 100 M)$ for $t=10^{4} \mathrm{M}$. The lowest mode rates in the odd modes (red dash-dotted on the right panel) are truncated due to numerical oscillations.

small solutions ([24,25]). For the dominant $\ell^{\prime}=\ell=0$ mode the estimate in standard Schwarzschild coordinates reads

$$
|\phi| \leq \frac{C_{1}}{\left(C_{2}+t+r_{*}\right)\left(C_{3}+t-r_{*}\right)^{2}},
$$

where $C_{i}$ are constants. This estimate captures the asymptotic behavior of the solution near null infinity as well as at finite distances from the black hole. It also provides an 
explicit description of the point- and slicing-dependence of the decay rates [18]. The powers in the above formula are different for different values of $\ell$, but the qualitative structure of observer-dependence is the same.

The far-field local-in-time decay rates suggested by Fig. 1 are listed in Tab. 1 Notice that in Tab. 1 the entries that were indeterminate in Tab. 1 of [15] are printed with an asterisk. We emphasize that these are the decay rates that would be observed by late-time observers who are not asymptotic. Initial data (compact support data centered at $r_{*}=25 M$ ) and observation points (far away observers with $r_{*} \geq 100 M$ ) were chosen here so that even very long evolutions do not reveal the inter-mode interaction effect in the near field to be discussed below in Sec. 3.2

\begin{tabular}{|c||cc|cc|cc|}
\hline$\ell^{\prime}$ & \multicolumn{2}{|c|}{$\ell=0$} & \multicolumn{2}{c|}{$\ell=2$} & \multicolumn{2}{c|}{$\ell=4$} \\
\hline \hline 0 & -2 & -3 & -4 & -5 & -6 & -7 \\
2 & -2 & -3 & -4 & $-7^{*}$ & -6 & $-9^{*}$ \\
4 & -4 & -5 & -4 & -7 & -6 & $-11^{*}$ \\
\hline
\end{tabular}

\begin{tabular}{|c||cc|cc|cc|}
\hline$\ell^{\prime}$ & \multicolumn{2}{|c|}{$\ell=1$} & \multicolumn{2}{c|}{$\ell=3$} & \multicolumn{2}{c|}{$\ell=5$} \\
\hline \hline 1 & -3 & -5 & -5 & -7 & -7 & -9 \\
3 & -3 & -5 & -5 & $-9 *$ & -7 & $-11^{*}$ \\
5 & -5 & -7 & -5 & -9 & -7 & $-13^{*}$ \\
\hline
\end{tabular}

Table 1 Far-field decay rates for all mode projections suggested by Fig. 1 for even modes (left table) and odd modes (right table). We list both $n_{\infty}^{\mathscr{I}}$ (left entry of each column) and $n_{\infty}^{i^{+}}$(right entry) for each choice of $\ell^{\prime}, \ell$. The values agree with theoretical predictions listed in (1) (see [5 12]). However, some of the finite distance rates, marked with an asterisk, may change asymptotically in time due to propagation of near-horizon inter-mode coupling behavior not seen in Fig. 1 because the initial data and the observers are located far away from the black hole (see Sec. 3.2.

\subsubsection{Local rates of the full field}

The 'splitting' in the decay rates of the full field has first been observed in [14]. We present in Fig. 2 the local decay rate for the full field for initial data of $\ell^{\prime}=4$ for a family of observers in linear (left panel) and inverse (right panel) time.

The curves separate into two types: those corresponding to near observers for whom the local decay rate at the end point of the numerical simulation increases as time increases (or as inverse time decreases), and those corresponding to far observers for whom it decreases in the same limits. Notice that the designations 'near' and 'far' here are different than in the preceding section. The designation of near or far observers depends on how long the simulation runs. For our choice of $t_{\text {final }}=10^{4} \mathrm{M}$, the two types of curves separate at about $r \sim 700 M$. For near observers $(r \lesssim 700 M)$ extrapolations of the local decay rate as a function of inverse time to $M / t \rightarrow 0$ produce results very close to the expected asymptotic value of -5 , but far away observers seem to measure a decay rate of -6 . Quantitatively, the observers and the extrapolated decay rates they measure are as follows: $\left(r_{*} / M, n_{\infty}^{i^{+}}\right)=\{(112,-4.962)$, (523, -5.008), (608, -5.121), (728, -5.017), (907,-5.058), (1207, -5.495),

$(1806,-6.08)\}$. The latter two values vary significantly from -5 , which is what is referred to as the full-field 'splitting.' Notice, however, that Fig.2 2also shows how the 'splitting' behavior is an intermediate one: the $\left(r_{*} / M, n_{\infty}^{i^{+}}\right)=(1207,-5.495)$ case shows that although the extrapolated value deviates significantly form the expected value of -5 , the curve already starts to curve up as $M / t$ decreases. The integration, 



Fig. 2 The local decay rates for $\ell^{\prime}=4$ initial data as functions of time $(t / M$, left panel) and inverse time ( $M / t$, right panel). The bold solid magenta curve corresponds to an observer at $r=700 M$, dashed blue curves for observers at $r>700 M$, and thin solid grin curves to observers at $r<700 M$. For the right panel, for high $M / t$ the distance of the observer increases from bottom to top. Note that the uppermost two thin solid green curves would appear below the thin solid green curves at the lower right corner were the figure extended to higher values of $M / t$. The dotted curves on the right panel are extrapolations of the local decay rates using linear extrapolation for $r<700 M$ and quadratic extrapolations for $r \geq 700 M$.

however, is not long enough to allow for sufficient curvature to enter the curve, that would push the asymptotic value all the way up to -5 . The situation would be the same if we only integrated any of the solid curves in Fig. 2 to earlier times. In the case of $\left(r_{*} / M, n_{\infty}^{i^{+}}\right)=(907,-5.058)$ the curve has enough to make the limit be reasonably close to -5 , and we conclude that that would also be the case for more distant observer, if we integrate longer in time.


Fig. 3 Absolute values of the scalar field for the evolution measured by observers along null infinity and at $r_{*}=200 M$ for each projected mode as functions of the time, for initial modes $\ell^{\prime}=0$ (left panel) and $\ell^{\prime}=4$ (right panel). For each case the steeper curve at late times corresponds to the field for the stationary observer at a finite distance. The lowest excited mode is the dominant mode. It is larger at all times by many orders of magnitude for $\ell^{\prime}=0$ whereas for $\ell^{\prime}=4$ the lowest excited mode dominates only at late times (see Fig. 4 for the ratio between the two lowest excited modes). The transition is observed as an intermediate 'splitting' in the full field. 


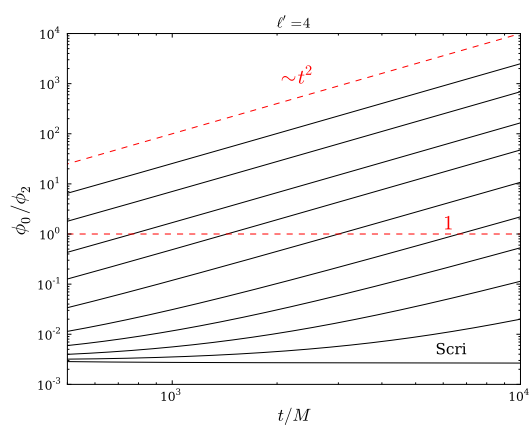

Fig. 4 Ratio of the lowest generated modes as a function of time. We plot the ratios of $\ell=0$ to $\ell=2$ modes for $\ell^{\prime}=4$ initial data measured by observers ranging from the vicinity of the horizon to null infinity (from top to bottom in the figure). Two dashed red reference lines indicate a ratio of unity, and the time development of the ratio as $t^{2}$ between the modes valid for observers close to the black hole. The plot shows that the lowest mode will dominate for any finite distance observer at sufficiently late times, but the notion of near and far observers depends on the evolution time. There is no transition of decay rates along null infinity, referred to as Scri in the above figure.

It may seem puzzling that the local decay rates do not present any 'splitting' behavior (Fig. 1) whereas the full field does (Fig. 2). This puzzle is explained by considering the relative strengths of modes generated due to mode coupling in Kerr spacetime. We plot in Fig. 3 the absolute values of the projected modes for initial data with $\ell^{\prime}=0$ on the left and $\ell^{\prime}=4$ on the right panel for comparison. The values are measured by two observers for each mode: one at null infinity, the other at $200 M$. The dominant modes for $\ell^{\prime}=0$ are by many orders of magnitude larger than the up-modes generated by mode coupling. This implies that the full field clearly decays with the rate of the dominant mode, and no 'splitting' occurs. This clean separation of absolute values in projected modes is no more valid for initial data with $\ell^{\prime}=4$ as seen in the right panel of Fig. 3. The initial mode starts strongest, but decays fast leaving the generated lower modes behind. The null infinity decay rates of the lowest two modes are the same, implying that there is no transition of strength between them (compare also Fig. 11. The finite distance rates of these modes, however, are different. Consequently, the lowest mode with slowest decay dominates at late times, but at early times the decay of the faster decaying higher mode may still dominate. This leads to the apparent 'splitting' behavior at far away distances indicated in Fig. 2 . We note that the same behavior is also found in the case of odd $\ell, \ell^{\prime}$ modes.

We argue that full-field 'splitting' appears because the simulation has not run far enough for far away observers. Longer evolutions would make far observers near ones. Considering Fig. 2. we predict that the asymptotic decay rates measured by far observers would be numerically close to -5 for longer evolutions. Notably, already with the given evolution time the extrapolations for the distant observers curve "up" in Fig. 2, in support of our prediction.

Further support for this argument comes from the ratio of the two lowest generated modes, $\phi_{\ell^{\prime \prime \prime}=0} / \phi_{\ell^{\prime \prime}=2}$ for $\ell^{\prime}=4$, plotted in Fig. 4. This plot gives additional information to the evolution of modes whose decay rates are depicted in Fig. 1. As 
can be inferred from Fig. 11, the rates along null infinity between the two lowest generated modes are the same. Therefore, their ratio stays below unity and no transition is observed for the decay along null infinity. For observers closer to the black hole (upper curves in the figure), however, the ratio evolves in time and goes above unity eventually. The dashed red line depicting a ratio of 1 can be seen as describing the transition of the decay rate from the rate of the higher mode to the lowest one. The fact that the ratio goes above unity for finite distance observers indicates that the lowest mode becomes the dominant mode whose decay is observed. The farther away the observer the longer it takes for the lowest generated mode to dominate. In that sense the 'splitting' of the decay rates for the full field is only an intermediate behavior due to the relative strength of generated modes. For observers near the black hole, the difference in the decay rates is 2 , so the ratio follows the $t^{2}$ curve depicted by the second dashed red line in Fig. 4.

\subsection{Near-field}

The picture of decay rates in certain projected modes presented in Fig. 1 1 and Tab. 1 is modified when near horizon rates are taken into account. Rácz and Tóth report 'splitting' in [15] $]^{1}$ in the projected modes for certain cases. We plot the near horizon decay rates for two cases, $\ell^{\prime}=\ell=2$ and $\ell^{\prime}=\ell=3$ in Fig. 5 on the left and the right panels respectively. The observers are located (from large distances to lower distances) at $r_{*} / M=20,18,16,14,12,10$ (yellow dashed), $r_{*} / M=8,6,4,2,0,-4$ (green solid) and $r_{*} / M=-8,-12,-20,-70,-\infty$ (blue dotted).

In such cases with $\ell^{\prime}=\ell$, the determination of the asymptotic decay rate is particularly difficult due to the near-field intermediate behavior. Decay rates along $r_{*} \geq 10 M$ denoted by the yellow dashed lines are consistent with Fig. 1 and Tab. 1 The local rate curves bend down, however, in preparation for a sign change, which happens along the green solid lines in the range $r_{*} \in[-4 M, 8 M]$. The rate after the sign change approaches the same rate as the blue dotted lines, plotted for observers with $r_{*} \leq-8 M$. The green vertical lines in Fig. 5 indicating sign change can be regarded as the boundary of 'splitting' between the two finite distance decay rates.

The location dependence observed in Fig. 5 5 provides evidence for the difference between intermediate and asymptotic behavior. We suggest that, asymptotically in time, the decay of the blue dotted lines in Fig. 55 will dominate for all finite observers but at any given late time there will be some far away observers that see the decay of the yellow dashed lines consistent with Tab. 1.

Given that the asymptotic behavior dominates for different observers at different times, a natural question is how the boundary between the two regimes (intermediate and asymptotic) propagates to infinity. We see from Fig. 5 that the boundary can be taken as where the sign of the field changes, depicted by the vertical green lines. The time difference between the vertical green lines seems to become larger for the farther away observers, indicating that the speed of transition slows down. We would like to know quantitatively, for a given observer, the speed of the transition. In Fig. 6, we

\footnotetext{
1 We thank Gábor Zs Tóth for pointing out that 'splitting' occurs near the horizon for the projected modes.
} 

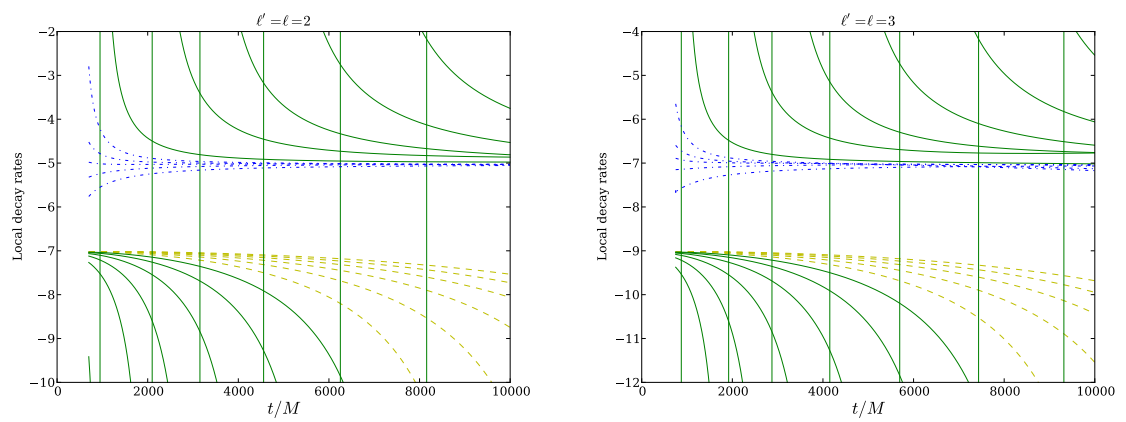

Fig. 5 Local decay rates for near-horizon observers with $\ell^{\prime}=\ell=2$ (left panel) and $\ell^{\prime}=$ $\ell=3$ (right panel). The observers are located (from large distances to lower distances) at $r_{*} / M=20,18,16,14,12,10$ (yellow dashed), $r_{*} / M=8,6,4,2,0,-4$ (green solid) and $r_{*} / M=$ $-8,-12,-20,-70,-\infty$ (blue dotted). The yellow dashed lines are consistent with Fig. 11 and Tab. 1 The green solid vertical lines indicate the transition during which the field changes sign. The blue dotted lines are the near-horizon rates. The plot indicates that at asymptotically late times the near-horizon rates (blue dotted) will dominate for far away observers (yellow dashed) after a sign change (green solid).

plot the rate at which this transition event moves as a function of the inverse distance to the observer for the case $\ell^{\prime}=2=\ell$. We show the rate for five sets of initial data characterized by the $r_{*}$ value of the center of the initial wave packet. Specifically, we set the centers of the initial wave packets as $r_{* 0} / M=5,10,15,20$ and 25 .

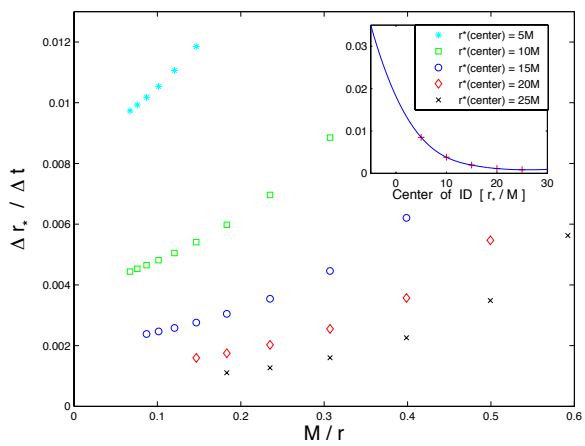

Fig. 6 Rate at which the boundary of the 'splitting' behavior moves as a function of the inverse distance of the observer. We show the rate for five sets of initial data characterized by the $r_{*}$ value of the center of the initial wave packet. Specifically, we choose $\ell^{\prime}=2=\ell$ and the centers of the initial wave packets are at $r_{* 0} / M=5,10,15,20$ and 25 . The insert shows the extrapolated value of the boundary speed at infinity $(M / r \rightarrow 0)$ as a function of the location of the center of the initial wave packet and a spline extrapolation thereof.

Near observers see a faster propagation speed of the boundary than distant observers. In addition, initial wave packets closer to the black hole cause a faster transition. Both properties indicate that the transition is a strong field behavior. Intuitively 
it makes sense that the asymptotic decay rate is obtained faster for all observers when the initial perturbation is in the strong field domain close to the black hole.

The insert shows the extrapolated value of the boundary speed at infinity $(M / r \rightarrow$ 0 ) as a function of the location of the center of the initial wave packet and a spline extrapolation thereof. Even for far observers, the transition speed seems to approach a non-vanishing limit. This dependence of the 'splitting' behavior on the location of the initial perturbation has not been observed before.

In conclusion, we distinguish late time behavior for large $t$ from asymptotic behavior for $t \rightarrow \infty$. In that sense, 'splitting' in the projected modes should be understood as an intermediate late time behavior, and not an asymptotic behavior.

Our explanation for this behavior is that successive mode excitations affect the decay rate of the late-time tail via inter-mode coupling. For example, the decay rate of the $\ell=4$ mode given $\ell^{\prime}=2$ initial data progresses in multiple channels. The direct channel is the excitation of the $\ell=4$ mode by the $\ell^{\prime}=2$ initial data. This direct channel leads to a tail with asymptotic decay rate of $t^{n_{0}}$ where $-n_{0}=\ell^{\prime}+\ell+3=$ $2+4+3=92$ In an indirect channel, the $\ell^{\prime \prime}=0$ is excited. The excited monopole field then acts as a source and creates a second $\ell=4$ tail whose asymptotic decay rate is riven by $t^{n_{1}}$ where $-n_{1}=\ell^{\prime \prime}+\ell+3=0+4+3=7$. Similarly, the decay rate of the $\ell=2$ mode given $\ell^{\prime}=2$ initial data progresses in multiple channels. In the direct channel, the $\ell=2$ mode evolves without coupling, for which the asymptotic decay rate is given by $t^{n_{0}}$ where $-n_{0}=\ell^{\prime}+\ell+3=2+2+3=7$. The indirect channel involves the excitation of the $\ell^{\prime \prime}=0$ mode, which then acts as a source field for a second $\ell=2$ field whose tail decays asymptotically according to $t^{n_{1}}$ where $-n_{1}=\ell^{\prime \prime}+\ell+3=0+2+3=5$. We propose that the reason why in [15] these values (and others) were indeterminate is that the multiple channels could not be computed. Note that these observations suggest that the tail decay rates found by Hod [5] are not asymptotic, because they ignore the inter-mode interaction. This reasoning also explains the $m$-dependence of the 'splitting' as observed in [15. 16]. We summarize our findings in Tab. 2. Notice how these values differ from their counterparts in Tab. 1. This intricate inter-mode coupling mechanism has not been suggested before, and warrants further study.

\begin{tabular}{|c||cc|cc|cc|}
\hline$\ell^{\prime}$ & \multicolumn{2}{|c|}{$\ell=0$} & \multicolumn{2}{c|}{$\ell=2$} & \multicolumn{2}{c|}{$\ell=4$} \\
\hline \hline 0 & -2 & -3 & -4 & -5 & -6 & -7 \\
2 & -2 & -3 & -4 & -5 & -6 & -7 \\
4 & -4 & -5 & -4 & -7 & -6 & -9 \\
\hline
\end{tabular}

\begin{tabular}{|c||cc|cc|cc|}
\hline$\ell^{\prime}$ & \multicolumn{2}{|c|}{$\ell=1$} & \multicolumn{2}{c|}{$\ell=3$} & \multicolumn{2}{c|}{$\ell=5$} \\
\hline \hline 1 & -3 & -5 & -5 & -7 & -7 & -9 \\
3 & -3 & -5 & -5 & -7 & -7 & -9 \\
5 & -5 & -7 & -5 & -9 & -7 & -11 \\
\hline
\end{tabular}

Table 2 Same as in Table 1 with the near-field effect of first-order inter-mode coupling.

Based on [35] we know that starting with a some value of $\ell^{\prime}$, all dynamically allowed lower modes are excited at higher orders in $(a / r)^{2}{ }^{3}$ Specifically, starting

\footnotetext{
2 In what follows we omit the subscript from the asymptotic decay rate $n_{\infty}$ as it is implied.

3 We use here the expansion in powers of $(a / r)^{2}$ not necessarily as an expansion in a small parameter as in [35, but ronoather as a counting scheme for the orders of the multiple channels; however, for small $a / r$ this also explains the smallness of the amplitude of the excited source.
} 
with an $\ell^{\prime}=4$ mode and considering the $\ell=4$ mode, we have the direct channel that leads to decay rate with $t^{n_{0}}$ where $-n_{0}=\ell^{\prime}+\ell+3=4+4+3=11$. We also have two indirect channels, one with the excited quadrupole mode and the other with the excited monopole mode. Naive application of our argument above would lead to decay rate with $t^{n_{1}}$ where $-n_{1}=\ell^{\prime \prime}+\ell+3=2+4+3=9$, and the latter the decay rate with $t^{n_{2}}$ where $-n_{2}=\ell^{\prime \prime \prime}+\ell+3=0+4+3=7$. If this reasoning were correct, one would expect the asymptotic decay rate to be $t^{-7}$. However, in practice we find the decay rate to be $t^{-9}$. We propose that this different decay rate is found because the produced monopole does not decay at the same rate that a monopole present in the initial data does. Indeed, the former decays as $t^{-3}$ whereas the latter decays as $t^{-5}$. This difference is proposed to be responsible for the faster decay rate we find. In the previously discussed case of $\ell^{\prime}=2, \ell=2$ the produced monopole decays at the same rate as a monopole present in the initial data is, and therefore the decay rate obeyed the naive expectations. See the discussion in [36].

It is challenging to observe such decay rates numerically due to the very high order effect that is needed to produce them. For the $\ell^{\prime}=4, \ell=4$ case and counting powers of $(a / r)^{2}$, the $\ell^{\prime \prime}=2$ mode is excited at $1^{\text {st }}$ order, i.e., at $O\left((a / r)^{2}\right)$, and the $\ell^{\prime \prime \prime}=0$ mode is excited at $2^{\text {nd }}$ order, i.e., at $O\left((a / r)^{4}\right)$. Continuing the counting of orders, $n_{1}$ is a $2^{\text {nd }}$ order effect at $O\left((a / r)^{4}\right)$, while $n_{2}$ is a $4^{\text {th }}$ order effect at $O\left((a / r)^{8}\right)$. Since each mode is excited with a small amplitude, higher order excitations are expected to be produced with very low amplitudes which set a considerable challenge for numerical computations because of the challenges involved with amplitudes comparable or smaller than the floating point arithmetic level, and the very long evolution times which are needed for very low initial amplitude to dominate despite their slower decay rate.

Numerical evidence for such higher order tails await further studies [36]. In the meantime, one may tentatively speculate on the basis of the above argument and our numerical results of Table 2 that the successive mode excitations ultimately impact the asymptotic decay rate of each and every mode. With the exception of the case in which $\ell^{\prime}$ is the slowest decaying mode (for which case the decay rates are given by $-n=\ell^{\prime}+\ell+3$ ), all other modes - even or odd - appear to decay according to $-n=\ell^{\prime}+\ell+1$.

\section{Summary and Conclusions}

We presented a detailed study of 'splitting' of local decay rates in Kerr spacetime observed in previous work $([14,15,16,23])$ both in certain projected modes and in the full field. Our simulations are about an order of magnitude longer $\left(\sim 10^{4} \mathrm{M}\right)$ than currently published simulations in the literature, which allows us to determine power indices that were considered, based on shorter evolutions, as ambiguous. The determination of these indices suggests a spin-dependent mechanism by which a 'splitting'-like behavior in the decay rates is produced. To achieve long simulation times without destroying relevant features of the solution due to boundary effects, we attached hyperboloidal layers both in the positive and the negative directions in the tortoise coordinate in Kerr spacetime. This procedure leads to a horizon-penetrating, 
hyperboloidal coordinate system, completely removing artificial boundaries from the numerical simulation (see also [33] for the construction in Schwarzschild spacetime). It is a new result that a hyperboloidal layer can be attached in the strong field domain near the future event horizon of Kerr spacetime, which may be of potential usefulness also for other applications. Some interesting problems where this method might be used are the study of superradiance (see, for example, recent work [37]), and the instability of perturbations of extremal Kerr spacetimes ([38]).

Having access to long simulations and studying various aspects of the solution from local decay rates to relative amplitudes of projected modes, we obtained a detailed understanding of the mechanisms underlying near-horizon and far-field 'splitting.' These two aspects of 'splitting' seem unrelated to each other. 'Splitting' near the black hole appears in the projected modes, and can be observed with standard codes. It is surprising that it has been discovered only recently. 'Splitting' far away appears in the full field and is due to the competition between the amplitudes of projected modes.

In cases where full-field 'splitting' appears (for example, Fig. 2) we observe that different modes have different decay rates. Eventually the lowest mode dominates because of its slowest decay (Fig. 3). This transition happens at different times for different observers, which appears as 'splitting' in the decay rates of the full field. Asymptotically in time, the theoretical decay rates are valid. In that sense, the 'splitting' of the full field is only an intermediate behavior.

'Splitting' in certain projected modes is of a different nature arising from the excitation of lower modes. Here we observe that for any given time there are three domains with three different local decay rates (see Figs. 11 and 5): very close to the horizon, far away from the horizon, and near infinity. There is a transition in which the field changes sign and the decay rates approach the near-horizon rates. The boundary of this transition moves slowly towards infinity (Fig. 6, which suggests that asymptotically in time near-horizon rates will dominate at all finite distances. For all practical purposes, however, 'splitting' is a real effect for these modes at any given (late) time. We also argued that successive mode excitations affect the decay rate of the late-time tail of all modes. However, the effects of lower mode excitations on any given mode's decay rate are difficult to observe due to numerical challenges. This is an area certainly requiring further work.

\section{Acknowledgments}

We thank Gábor Zs Tóth and István Rácz for discussions. AZ is supported by the NSF Grant PHY-1068881, and by a Sherman Fairchild Foundation grant to Caltech. GK acknowledges research support from NSF Grant Nos. PHY-1016906, PHY-113566 and PHY-1303724. LMB is supported by a NASA EPSCoR RID grant and by NSF grants PHY-0757344, PHY-1249302, DUE-0941327 and DUE-1300717. Initial work on this project was done while LMB was at the University of Alabama in Huntsville. Most of the data presented in this work were generated on the Air Force Research Laboratory CONDOR supercomputer. GK also acknowledges support from AFRL under CRADA No. 10-RI-CRADA-09. 


\section{References}

1. T. Regge, J.A. Wheeler, Phys.Rev. 108, 1063 (1957). DOI 10.1103/PhysRev.108.1063

2. R.H. Price, Phys.Rev. D5, 2419 (1972). DOI 10.1103/PhysRevD.5.2419

3. L. Barack, Phys.Rev. D61, 024026 (2000). DOI 10.1103/PhysRevD.61.024026

4. L. Barack, A. Ori, Phys.Rev.Lett. 82, 4388 (1999). DOI 10.1103/PhysRevLett.82.4388

5. S. Hod, Phys. Rev. D61, 024033 (1999). DOI 10.1103/PhysRevD.61.024033

6. S. Hod, Physical Review D 61, 064018 (2000)

7. E. Poisson, Phys.Rev. D66, 044008 (2002). DOI 10.1103/PhysRevD.66.044008

8. W. Krivan, Phys.Rev. D60, 101501 (1999). DOI 10.1103/PhysRevD.60.101501

9. L.M. Burko, G. Khanna, Phys.Rev. D67, 081502 (2003). DOI 10.1103/PhysRevD.67.081502

10. M. Tiglio, L.E. Kidder, S.A. Teukolsky, Class.Quant.Grav. 25, 105022 (2008). DOI 10.1088/ 0264-9381/25/10/105022

11. L.M. Burko, G. Khanna, Class.Quant.Grav. 26, 015014 (2009). DOI 10.1088/0264-9381/26/1/015014

12. L.M. Burko, G. Khanna, Class.Quant.Grav. 28, 025012 (2011). DOI 10.1088/0264-9381/28/2/025012

13. C. Gundlach, R.H. Price, J. Pullin, Phys.Rev. D49, 883 (1994). DOI 10.1103/PhysRevD.49.883

14. A. Zenginoglu, M. Tiglio, Phys. Rev. D80, 024044 (2009). DOI 10.1103/PhysRevD.80.024044

15. I. Racz, G.Z. Toth, Class.Quant.Grav. 28, 195003 (2011). DOI 10.1088/0264-9381/28/19/195003

16. M. Jasiulek, Class.Quant.Grav. 29, 015008 (2012). DOI 10.1088/0264-9381/29/1/015008

17. M. Purrer, S. Husa, P.C. Aichelburg, Phys.Rev. D71, 104005 (2005). DOI 10.1103/PhysRevD.71. 104005

18. A. Zenginoglu, Class. Quant. Grav. 25, 175013 (2008). DOI 10.1088/0264-9381/25/17/175013

19. F. Pretorius, W. Israel, Class. Quant. Grav. 15, 2289 (1998). DOI 10.1088/0264-9381/15/8/012

20. S.J. Fletcher, A.W.C. Lun, Class. Quant. Grav. 20, 4153 (2003)

21. L.R. Venter, N.T. Bishop, Phys. Rev. D73, 084023 (2006). DOI 10.1103/PhysRevD.73.084023

22. A. Zenginoglu, Class. Quant. Grav. 25, 145002 (2008). DOI 10.1088/0264-9381/25/14/145002

23. E. Harms, S. Bernuzzi, B. Bruegmann, Class.Quant.Grav. 30, 115013 (2013). DOI 10.1088/ 0264-9381/30/11/115013

24. W. Strauss, K. Tsutaya, Discr. Cont. Dynamical Systems 3, 175 (1997)

25. N. Szpak, P. Bizon, T. Chmaj, A. Rostworowski, J.Hyperbol.Diff.Equat. 6, 107 (2009)

26. B.B. E. Harms, S. Bernuzzi, Class. Quantum Grav. 30, 115013 (2013)

27. Y. Mino, M. Sasaki, T. Tanaka, Phys.Rev. D55, 3457 (1997). DOI 10.1103/PhysRevD.55.3457

28. T.C. Quinn, R.M. Wald, Phys.Rev. D56, 3381 (1997). DOI 10.1103/PhysRevD.56.3381

29. A. Zenginoglu, C.R. Galley, Phys.Rev. D86, 064030 (2012). DOI 10.1103/PhysRevD.86.064030

30. M. Casals, S. Dolan, A.C. Ottewill, B. Wardell, Phys.Rev. D88, 044022 (2013). DOI 10.1103/ PhysRevD.88.044022

31. A. Zenginoglu, J.Comput.Phys. 230, 2286 (2011). DOI 10.1016/j.jcp.2010.12.016

32. A. Zenginoglu, G. Khanna, Phys.Rev. X1, 021017 (2011). DOI 10.1103/PhysRevX.1.021017

33. S. Bernuzzi, A. Nagar, A. Zenginoglu, Phys.Rev. D86, 104038 (2012). DOI 10.1103/PhysRevD.86. 104038

34. L.M. Burko, A. Ori, Phys.Rev. D56, 7820 (1997). DOI 10.1103/PhysRevD.56.7820

35. R. Gleiser, R. Price, J. Pullin, Class.Quant.Grav 25, 072001 (2008)

36. L.M. Burko, G. Khanna, (2013)

37. P. Csizmadia, A. Laszlo, I. Racz, Class.Quant.Grav. 30, 015010 (2013). DOI 10.1088/0264-9381/30/ $1 / 015010$

38. S. Aretakis, (2012) 